\title{
Generic emergence of objectivity of observables in infinite dimensions
}

\author{
Paul A. Knott, ${ }^{1, *}$ Tommaso Tufarelli, ${ }^{1}$ Marco Piani, ${ }^{2}$ and Gerardo Adesso ${ }^{1}$ \\ ${ }^{1}$ Centre for the Mathematics and Theoretical Physics of Quantum Non-Equilibrium Systems (CQNE), \\ School of Mathematical Sciences, University of Nottingham, University Park, Nottingham NGr 2RD, UK \\ ${ }^{2}$ SUPA and Department of Physics, University of Strathclyde, Glasgow, G4 ONG, UK
}

(Dated: September 29, 2018)

\begin{abstract}
Quantum Darwinism posits that information becomes objective whenever multiple observers indirectly probe a quantum system by each measuring a fraction of the environment. It was recently shown that objectivity of observables emerges generically from the mathematical structure of quantum mechanics, whenever the system of interest has finite dimensions and the number of environment fragments is large [F. G. S. L. Brandão, M. Piani, and P. Horodecki, Nature Commun. 6, 7908 (2015)]. Despite the importance of this result, it necessarily excludes many practical systems of interest that are infinite-dimensional, including harmonic oscillators. Extending the study of Quantum Darwinism to infinite dimensions is a nontrivial task: we tackle it here by using a modified diamond norm, suitable to quantify the distinguishability of channels in infinite dimensions. We prove two theorems that bound the emergence of objectivity, first for finite mean energy systems, and then for systems that can only be prepared in states with an exponential energy cut-off. We show that the latter class includes any bounded-energy subset of single-mode Gaussian states.
\end{abstract}

How does the objective classical world emerge from an underlying quantum substrate? The theories of decoherence and Quantum Darwinism (QD) can provide a rigorous framework to answer this question [1-7]. Firstly, in decoherence we acknowledge that realistic systems are rarely isolated, but rather are coupled to an inaccessible environment. It can then be shown that, under suitable assumptions on the system-environment interaction, only states of a certain basis - the pointer basis - survive the system-environment interaction, while superpositions of these pointer states are suppressed. QD extends this by considering observers who interact indirectly with the system by having access to fragments of the environment, as illustrated in Fig. 1: each observer measuring the system only has access to a single fragment of the environment (for example, we observe everyday objects by measuring a small fragment of the vast photon environment). This formalism can now be used to prove the emergence of objectivity: it has been shown, using various models [8-19], that multiple observers measuring the same system will invariably agree on the outcomes of their measurements, and hence their outcomes can be regarded as objective.

To obtain a complete description of objectivity, we must also describe how the objectivity of observables emerges - namely, why do multiple observers measure the same observables? Indeed, if different observers had access to inequivalent observables, the very notion of objectivity of the measurement outcomes would be illdefined. Objectivity of observables has been studied in numerous contexts, where it was shown that, when an observer only has access to a single fragment of the environment, the only information available is information about certain preferred observables, which correspond to

\footnotetext{
*Paul.Knott@nottingham.ac.uk
}

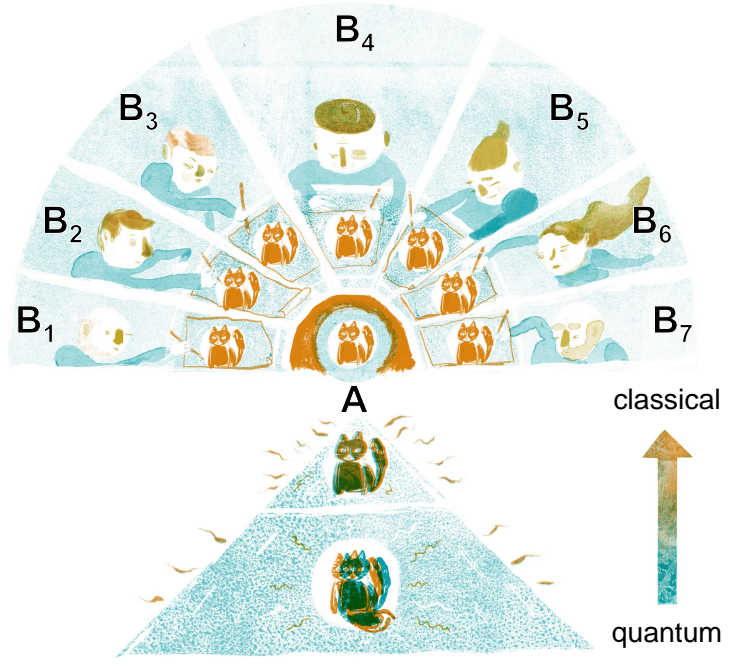

FIG. 1. In Quantum Darwinism, the objective classical reality (image of a cat, subsystem $A$ in the middle) emerges from an underlying quantum mechanical description (bottom layer, illustrating superposition effects) through the observation of multiple environment fragments (subsystems $B_{1} \ldots B_{N}$, depicted as painting artists). Here we show that the objectivity of observables is generic even when $A$ is an infinitedimensional quantum system, subject to suitable energy constraints. Artwork by Joseph Hollis.

the preferred basis [3-19]. Until recently, the majority of research consisted of studying specific models; the question then remained of whether the emergence of objectivity is a generic feature, or only a model-specific one.

This changed with a result by Brandão et al. [20], who showed that objectivity of observables is a generic phenomenon that emerges in a model-independent way from the basic mathematical structure of quantum mechanics, whenever the number of environment fragments gets large. Objectivity was there intended in the sense 
of information available and agreed upon about some general measurement performed indirectly on the system and described by a positive-operator-valued measure (POVM) [21]. However, there was one caveat: the authors of [20] required the system of interest to live in a finite-dimensional Hilbert space. Despite the appeal of finite-dimensional results, they do not cover the physically very relevant case of continuous-variable systems.

In this Letter we overcome this significant restriction to prove that infinite-dimensional systems, under appropriate constraints, also exhibit objectivity of observables. Specifically, we show that objectivity emerges generically when considering either of the following physically motivated restrictions: (i) systems with finite mean energy which arguably include all realistic systems of interest; and (ii) systems with an exponential energy cut-off which include systems prepared in single-mode bosonic Gaussian states. In both cases, we prove exact bounds to show that, as the number of environment fragments grows large, objectivity of observables emerges. In [20], the bound on objectivity depended only on the system dimension and the number of environment fragments; in contrast, our bounds provide non-trivial extensions that show an explicit dependence on the system's mean energy in the first case, and on the strength of the exponential cut-off in the second. Our results rely on a combination of mathematical techniques of potential independent interest, and overall shed further light on the underlying structure of our physical reality.

As shown in Fig. 1, the framework we study consists of a collection of (generally infinite-dimensional) subsystems. We can select any one of these as our system of interest and label it $A$; the rest of the subsystems, denoted $B_{1}$ to $B_{N}$, are then taken to be the $N$ different fragments of the environment of $A$. We then consider any completely positive trace-preserving (CPTP) map, i.e., any quantum channel, $\Lambda: \mathcal{D}(A) \rightarrow \mathcal{D}\left(B_{1} \otimes \ldots \otimes B_{N}\right)$ from the system to the environments; here $\mathcal{D}(X)$ is the space of density matrices of system $X$. The next step is crucial to QD [3]: we assume that each observer who wishes to measure system $A$ can only do so by measuring one fragment of the environment. To model this, we define the channel $\Lambda_{j}:=\operatorname{Tr}_{\backslash B_{j}} \circ \Lambda$ as the effective dynamics from $A$ to $B_{j}$, where $\operatorname{Tr}_{\backslash B_{j}}$ indicates the partial trace over all fragments except $B_{j}$.

As anticipated, we shall consider restrictions on the properties of system $A$, but notice that throughout this letter the fragments $B_{1}, \ldots, B_{N}$ do not need to satisfy any constraint. Our main results are expressed through generalizations of the so-called diamond norm. The latter encapsulates the notion of best possible distinguishability between two different physical processes, as allowed by quantum mechanics, and does not take into account additional physical constraints, e.g. on the average energy involved in the discrimination procedure. The generalizations we will consider will instead consider said restrictions, e.g., focusing on systems with bounded mean energy. Specifically, we define (see also [22-24]):
Definition 1. (Energy-constrained diamond norm) For a Hermiticity-preserving linear map $\Lambda: \mathcal{D}(A) \rightarrow \mathcal{D}(B)$, and a finite $\bar{n}>0$, we define

$$
\|\Lambda\|_{\diamond \bar{n}}:=\sup _{\rho: \operatorname{Tr}\left(\rho \hat{n}_{A}\right) \leq \bar{n}}\left\|\Lambda_{A} \otimes \operatorname{id}_{C}[\rho]\right\|_{1}
$$

where $C$ is an arbitrary system, $\hat{n}_{A}$ is the number operator only for subsystem $A$ [25], and the supremum is calculated over all physical states $\rho$ of AC such that the energy of the reduced state $\rho_{A}$ respects the indicated bound.

In the above, we indicate with $\|X\|_{1}$ the trace norm of an operator. Given two states $\sigma_{0}$ and $\sigma_{1}$ of a system $S$, the trace norm of their difference, $\left\|\sigma_{0}-\sigma_{1}\right\|_{1}$, is directly linked to the ability to discriminate whether $S$ was prepared in either $\sigma_{0}$ or $\sigma_{1}$ [21]. The meaning of the energyconstrained diamond norm is then that of providing a measure of distinguishability of two evolutions $\Lambda_{0}$ and $\Lambda_{1}$, by considering in the above definition $\Lambda=\Lambda_{0}-\Lambda_{1}$ and an input state $\rho$ that has limited mean energy. Based on this definition, we can prove the following:

Theorem 1. Let $\Lambda: \mathcal{D}(A) \rightarrow \mathcal{D}(B)$ be a CPTP map. Define $\Lambda_{j}:=\operatorname{Tr}_{\backslash B_{j}} \circ \Lambda$ as the effective dynamics from $\mathcal{D}(A)$ to $\mathcal{D}\left(B_{j}\right)$ and fix a number $0<\delta<1$. Then there exists a POVM $\left\{M_{k}\right\}$ and a set $S \subseteq\{1, \ldots, N\}$ with $|S| \geq$ $(1-\delta) N$ such that, for all $j \in S$ (and for some finite mean energy $\bar{n})$, we have

$$
\left\|\Lambda_{j}-\mathcal{E}_{j}\right\|_{\diamond \bar{n}} \leq \frac{17}{\delta}\left(\frac{2}{7}\right)^{\frac{14}{17}}\left(\frac{\bar{n}^{7}}{N}\right)^{\frac{1}{17}} \approx \frac{6.06}{\delta}\left(\frac{\bar{n}^{7}}{N}\right)^{\frac{1}{17}}
$$

where the measure-and-prepare channel $\mathcal{E}_{j}$ is given by

$$
\mathcal{E}_{j}(X):=\sum_{k} \operatorname{Tr}\left(M_{k} X\right) \sigma_{j, k}
$$

for states $\sigma_{j, k} \in \mathcal{D}\left(B_{j}\right)$. Here both spaces of $A$ and $B$ can have infinite dimensions.

We prove this result (mean energy bound for brevity) in the Supplemental Material [26], focusing here on its interpretation. As explained in introducing the energyconstrained diamond norm, the LHS of Eq. (2) is a measure of how well the two channels $\Lambda_{j}$ and $\mathcal{E}_{j}$ can be distinguished. We see that, for a fixed fraction $1-\delta$ of the environment fragments, and fixed bound $\bar{n}$ on the average input energy, any quantum channel from $A$ to $B_{j}$ becomes arbitrarily close to the channel $\mathcal{E}_{j}$. The latter is known as a measure-and-prepare channel (or an entanglement-breaking channel [36]): it can be implemented by first measuring the system with the POVM $\left\{M_{k}\right\}$, and then preparing a state $\sigma_{j, k}$ that depends on the outcome obtained. Crucially, the measured observable is the same for all these environment fragments and hence is objective - any observer who wishes to probe system $A$ by measuring a fragment $B_{j}(j \in S)$ can at most gain information about the single POVM $\left\{M_{k}\right\}$. 


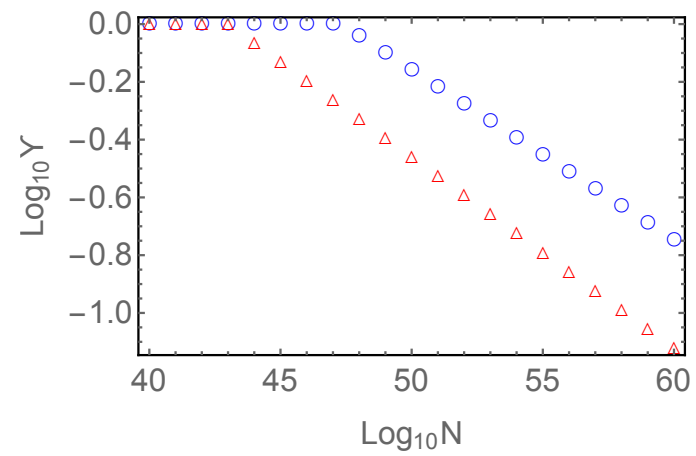

FIG. 2. (Color online) The number of environment fragments, $N$ (horizontal axis, logarithmic scale), is plotted against the upper bound on $\left\|\Lambda_{j}-\mathcal{E}_{j}\right\|_{\diamond \bar{n}}$, denoted $\Upsilon$ (vertical axis, logarithmic scale), which expresses the ability to discriminate between the channel $\Lambda_{j}=\operatorname{Tr}_{\backslash B_{j}} \circ \Lambda$ and the measure-andprepare channel $\mathcal{E}_{j}$, as given in Theorem 1 . Here we take $\bar{n}=1$ and $\delta=0.01$. We include the analyical bound in Eq. (2) (blue circles) and a numerically optimized bound (red triangles, see Supplemental Material [26]). A power-law fit of the numerical bound provides $\left\|\Lambda_{j}-\mathcal{E}_{j}\right\|_{\diamond \bar{n}} \leq \frac{1}{\delta} \beta N^{-\frac{1}{\alpha}}$ with $\beta \simeq 6.94, \alpha \simeq 15.18$. All plotted quantities are dimensionless.

Note that $\delta$ can be set as close to 0 as required, but this in turn affects the RHS and hence the minimum threshold in $N$ to provide a meaningful bound.

The RHS of Eq. (2), however, tends to zero very slowly with $N$, and therefore a huge number of environment fragments are needed to give an informative result. We illustrate this in Fig. 2, where we set $\bar{n}=1$ and $\delta=0.01$ (i.e. we ask whether objectivity of observables holds for $99 \%$ of the observers). We note that the unfavourable $\propto N^{-1 / 17}$ scaling can be improved slightly: in order to obtain a neat analytical result for Theorem 1 some approximations were taken, but a tighter bound can be found numerically (see Supplemental Material [26]). For the case $\bar{n}=1$, for example, we numerically obtain a scaling closer to $\propto N^{-1 / 15}$. Yet, even with this improved result, the upper bound of $\left\|\Lambda_{j}-\mathcal{E}_{j}\right\|_{{ }_{\diamond}}$ remains of the order of 0.1 when $N=10^{60}$.

Bounded mean energy states arguably include all physically realizable states of a continuous-variable system (which we assume can be written in a single-mode Fock basis) $[37,38]$. Despite this, due to the unfavourable scaling of the bound with the number $N$ of environments, Theorem 1 is of little practical use to show that objectivity of observables is generic in all such cases. To obtain a more informative bound, we can restrict further the class of states that are allowed in our diamond norm definition. In the following we will see that a more informative bound, admitting a vastly improved scaling with $N$, can be proved under such a restriction. The class of states we shall consider contains all the continuous-variable density matrices with an exponential energy cut-off in subsystem $A$. Specifically, we restrict to all density matrices $\rho_{A C}$ such that

$$
\operatorname{Tr}\left[\rho e^{\omega \hat{n}_{A}}\right] \leq \Omega,
$$

where $\Omega>1$ and $\omega>0$ are given constants. We show below that the exponential cut-off states include meaningful subsets of single-mode bosonic Gaussian states, which play a focal role in continuous-variable quantum information [39]. It is also trivial to show that any subset of states that can be written as a finite expansion in the Fock basis (up to some upper state $\left|n_{\max }\right\rangle$ ) belongs to this class. In passing, we note that it is in principle possible to consider even more general restrictions of the form $\operatorname{Tr}\left(f\left(\hat{n}_{A}\right) \rho\right) \leq \Omega$, where $f$ is a suitably chosen function. This should in turn allow us to recover the existing results for systems with a finite dimension $d[20]$ as a special instance, e.g. by choosing $f(n)=1$ for $n \leq d$, and $f(n)=e^{\omega n}$ for $n>d$, later taking the limit $\omega \rightarrow \infty$. However, such a generalization goes beyond the scope of this paper, and will be presented in a future publication.

We can then define another variant of the diamond norm, relevant when only states obeying Eq. (4) may be exploited to distinguish between channels.

Definition 2. (Exponential cut-off diamond norm) For a Hermiticity-preserving linear map $\Lambda: \mathcal{D}(A) \rightarrow \mathcal{D}(B)$, and constants $\omega>0, \Omega>1$, let

$$
\|\Lambda\|_{\diamond \omega, \Omega}:=\sup _{\operatorname{Tr}\left[\rho e^{\omega \hat{n}_{A}}\right] \leq \Omega}\left\|\Lambda_{A} \otimes \operatorname{id}_{C}[\rho]\right\|_{1} .
$$

where $C$ is an arbitrary ancillary system, $\hat{n}_{A}$ is the number operator only for subsystem $A$, and the supremum is calculated over all physical states $\rho$ of AC such that the reduced state $\rho_{A}$ respects the indicated bound.

Using this definition, we can now show the following:

Theorem 2. Let $\Lambda: \mathcal{D}(A) \rightarrow \mathcal{D}(B)$ be a CPTP map. Define $\Lambda_{j}:=\operatorname{Tr}_{\backslash B_{j}} \circ \Lambda$ as the effective dynamics from $\mathcal{D}(A)$ to $\mathcal{D}\left(B_{j}\right)$ and fix a number $0<\delta<1$. Then there exists a POVM $\left\{M_{k}\right\}$ and a set $S \subseteq\{1, \ldots, N\}$ with $|S| \geq$ $(1-\delta) N$ such that, for all $j \in S$ (and for some finite $\omega>0$ and $\Omega>1$ ), we have

$$
\left\|\Lambda_{j}-\mathcal{E}_{j}\right\|_{\diamond \omega, \Omega} \leq \frac{8}{\delta}\left(\frac{\gamma_{1}}{N}\right)^{1 / 3}\left[1+\frac{1}{4}\left(\ln \left(\gamma_{2} N\right)\right)^{4 / 3}\right],
$$

where the measure-and-prepare channel $\mathcal{E}_{j}$ is given in Theorem 1 and where

$$
\begin{aligned}
& \gamma_{1}=\frac{2 \tilde{d}^{2} s}{3 \omega^{4}}, \quad \gamma_{2}=\frac{3 \tilde{d} \omega^{4}}{16 s}, \quad \tilde{d}=\frac{\Omega e^{\omega}}{e^{\omega}-1}, \\
& \tilde{n}=\frac{1}{e^{\omega}-1}, \quad s=(\tilde{n}+1) \ln (\tilde{n}+1)-\tilde{n} \ln \tilde{n} .
\end{aligned}
$$

We prove this in the Supplemental Material [26]. Theorem 2 can be interpreted similarly to Theorem 1: with increasing $N$, provided the available resources obey the exponential cut-off condition, any quantum channel from $A$ to a generic environment fragment $B_{j}(j \in S)$ becomes 
arbitrarily close to the measure-and-prepare channel specified by the measurement of $\left\{M_{k}\right\}$ (again, the same measurement for all $j^{\prime}$ s). Let us remark, however, that the dominant scaling with $N$ is now $\propto(\ln N)^{4 / 3} / N^{1 / 3}$, which for $N \rightarrow \infty$ converges to zero significantly faster than the RHS of Eq. (2). It is instructive also to compare our results to the results in [20]: their bound scales as $\propto 1 / N^{1 / 3}$ and therefore approaches 0 faster than our bounds in the limit $N \rightarrow \infty$. However, with a straightforward modification of the proof of Theorem 2 we can derive a new bound applicable to large (yet finite) dimensions, under the further assumption of an exponential energy cut-off as in Eq. (4). Such a bound can potentially be more informative than the one from [20] at finite (but still large) values of $N$, if the system satisfies Eq. (4) with suitable values of $\omega, \Omega$.

It is important at this point to provide relevant examples of quantum states that satisfy the exponential energy cut-off condition. Let us consider the case in which the reduced density matrix of $A$ is an arbitrary mixed singlemode Gaussian state $\rho_{G}$ [39], specified by a displacement vector $\mathbf{d}=\sqrt{2}\{\Re(\alpha), \Im(\alpha)\}$ with $\alpha \in \mathbb{C}$, and by a covariance matrix $\mathbf{V}=\operatorname{diag}\left\{e^{2 r}(2 m+1), e^{-2 r}(2 m+1)\right\}$, where $m \geq 0$ is the mean number of thermal photons and we can fix without any loss of generality a real squeezing parameter $r>0$. Note that we may assume the covariance matrix to be in diagonal form, since diagonalization can always be achieved via a phase rotation commuting with $\hat{n}$. By means of the Husimi function $Q(\beta)=\pi^{-1}\langle\beta|\rho| \beta\rangle$, where $\{|\beta\rangle\}$ with $\beta \in \mathbb{C}$ is the overcomplete generating set of coherent states, we can evaluate the LHS of Eq. (4) analytically, using the formula $\operatorname{Tr}\left[\rho e^{\omega \hat{n}_{A}}\right]=e^{-\omega} \int_{\mathbb{C}} d^{2} \beta Q(\beta) e^{\left(1-e^{-\omega}\right)|\beta|^{2}}$, which may be derived by anti-normally ordering the operator $e^{\omega \hat{n}_{A}}[40]$. We then find that a Gaussian state $\rho_{G}$ satisfies the exponential cut-off condition if and only if

$$
\left\langle e^{\omega \hat{n}}\right\rangle=\frac{2 \exp \left[\frac{2 \Re(\alpha)^{2}}{\kappa_{+}^{2}}+\frac{2 \Im(\alpha)^{2}}{\kappa_{-}^{2}}\right]}{\left(e^{\omega}-1\right) \kappa_{+} \kappa_{-}} \leq \Omega
$$

with $\kappa_{ \pm}=\sqrt{\operatorname{coth}\left(\frac{\omega}{2}\right)-(2 m+1) e^{ \pm 2 r}}$. In the Supplemental Material [26], we exploit this formula to show that any subset of Gaussian states with bounded energy, i.e. $\mathcal{G}_{\bar{n}}=\left\{\rho_{G} \mid \operatorname{Tr}\left[\rho_{G} \hat{n}\right] \leq \bar{n}\right\}$, obeys the desired cut-off condition whenever the parameters $\omega, \Omega$ satisfy

$$
\begin{aligned}
& \Omega>1 /(1-\epsilon), \\
& \omega=\min \left\{\frac{2 \epsilon}{3 / 2+2 \bar{n}(2+\bar{n})}, \frac{1-\epsilon}{\bar{n}} \ln ((1-\epsilon) \Omega)\right\},
\end{aligned}
$$

where $0<\epsilon<1$ is an arbitrary parameter that can be tuned to optimize the resulting exponential cut-off bound. Note also that, once the relevant parameters have been fixed according to the above discussion, the entire convex hull of $\mathcal{G}_{\bar{n}}$ will also satisfy the exponential cut-off condition. For example, suppose one would like to distinguish between $\Lambda_{j}$ and $\mathcal{E}_{j}$, only being able to

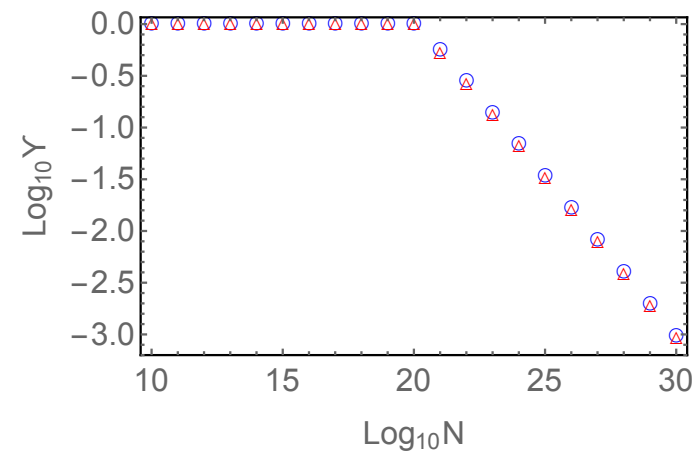

FIG. 3. (Color online) The number of environment fragments, $N$ (horizontal axis, logarithmic scale), is plotted against the upper bound on $\left\|\Lambda_{j}-\mathcal{E}_{j}\right\|_{\diamond \omega, \Omega}$, denoted $\Upsilon$ (vertical axis, logarithmic scale), given in Theorem 2. We take $\delta=0.01$ as in Fig. 2. The parameters $\omega, \Omega$ are optimized for each $N$ to provide the best possible bound attainable through our methods, assuming a set of Gaussian resource states $\mathcal{G}_{\bar{n}}$ with $\bar{n}=1$ (these would be the input states that can be used to discriminate between channels). Blue circles indicate the RHS of Eq. (6), while red triangles refer to a numerically optimized bound (see Supplemental Material [26]). A powerlaw fit of the latter yields $\left\|\Lambda_{j}-\mathcal{E}_{j}\right\|_{\diamond \omega, \Omega} \leq \frac{1}{\delta} \beta N^{-\frac{1}{\alpha}}$ with $\beta \simeq 5839, \alpha \simeq 3.20$. All plotted quantities are dimensionless.

prepare mixtures of Gaussians with $\left\langle\hat{n}_{A}\right\rangle \leq 1$. Then the results of Theorem 2 (exponential cut-off bound for brevity) would apply, giving us much tighter constraints on the emergence of objectivity as compared to what Theorem 1 would tell us under the same hypothesis. Furthermore, also in this case we can consider a numerical optimization yielding an improved upper bound for the RHS of Eq. (6) - see Supplemental Material [26]. This is shown in Fig. 3, where fixing $\delta=0.01$ and $\bar{n}=1$, as before, we obtain $\left\|\Lambda_{j}-\mathcal{E}_{j}\right\|_{\diamond \omega, \Omega}<0.5 \times 10^{-3}$ already for $N=10^{29}$.

It is remarkable that Theorems 1 and 2 hold for any channel $\Lambda$. These results, together with [20], show that objectivity of observables is built into the basic mathematical structure of quantum mechanics. Despite this, our analysis suggests that objectivity of observables may emerge extremely slowly in the absence of further restrictions on the system's properties and/or the form of its interaction with the environment: generally a vast number of environment fragments is needed to have truly informative bounds. This might simply be due to our energy-based bounds not being tight, and indeed future work should go towards improving such bounds. There are nonetheless a number of other directions for future studies.

Firstly, following our approach, further restrictions can be placed on the set of states defining the generalized diamond norms. A bound specifically for Gaussian states would be an interesting next step, as would be a bound that combines [20] with our results to consider, for example, finite-dimensional systems with a fixed energy. It 
would also be instructive to consider which states are responsible for allowing the channels in Theorems 1 and 2 to be easily distinguished. Do we expect these states to be realistic, experimentally producible states? Alternatively, restrictions can be placed on the measurements available to distinguish between the channels; considering only coarse-grained measurements would bring us closer to real-world scenarios where objectivity emerges.

A different line of attack would be to place restrictions on the allowed channels. One of the big questions that quantum Darwinism addresses is: why does our macroscopic every-day world appear objective and classical, despite being constructed of quantum mechanical particles? With this in mind we may ask: What general properties are shared by physically meaningful channels? If all relevant interactions between system and environment are ultimately due to, for example, a combination of one- and two-body Hamiltonians, the resulting channels may display some nontrivial structure. Less ambitiously, one may place further intuitive restrictions on the channels, such as the conservation of a global number operator of the form $\hat{n}_{A}+\hat{n}_{B_{1}}+\ldots+\hat{n}_{B_{N}}$ or other symmetry constraints. By investigating these generalizations of our results, it may be possible to further clarify why our macroscopic world is "classical", without resorting to the task of constructing macroscopic models and simulations, the like of which will only be possible with macroscopic-scale quantum computers.

\section{ACKNOWLEDGMENTS}

We thank Joseph Hollis for the artwork featured in Figure 1. We acknowledge discussions with F. G. S. L. Brandão, L. Lami, and A. Winter. This work was supported by the Foundational Questions Institute (fqxi.org) under the Physics of the Observer Programme (Grant No. FQXi-RFP-1601), the European Research Council (ERC) under the Starting Grant GQCOP (Grant No. 637352), and the European Unions Horizon 2020 Research and Innovation Programme under the Marie Skodowska-Curie Action OPERACQC (Grant Agreement No. 661338). T.T. acknowledges financial support from the University of Nottingham via a Nottingham Research Fellowship.
[1] W. H. Zurek, "Decoherence, einselection, and the quantum origins of the classical," Rev. Mod. Phys. 75, 715 (2003).

[2] M. Schlosshauer, "Decoherence, the measurement problem, and interpretations of quantum mechanics," Rev. Mod. Phys. 76, 1267 (2005).

[3] W. H. Zurek, "Quantum darwinism," Nature Phys. 5, 181 (2009).

[4] H. Ollivier, D. Poulin, and W. H. Zurek, "Objective properties from subjective quantum states: Environment as a witness," Phys. Rev. Lett. 93, 220401 (2004).

[5] H. Ollivier, D. Poulin, and W. H. Zurek, "Environment as a witness: Selective proliferation of information and emergence of objectivity in a quantum universe," Phys. Rev. A 72, 042113 (2005).

[6] R. Blume-Kohout and W. H. Zurek, "Quantum darwinism: Entanglement, branches, and the emergent classicality of redundantly stored quantum information," Phys. Rev. A 73, 062310 (2006).

[7] R. Horodecki, J. K. Korbicz, and P. Horodecki, "Quantum origins of objectivity," Phys. Rev. A 91, 032122 (2015).

[8] R. Blume-Kohout and W. H. Zurek, "Quantum darwinism in quantum brownian motion," Phys. Rev. Lett. 101, 240405 (2008).

[9] M. Zwolak, H. T. Quan, and W. H. Zurek, "Quantum darwinism in a mixed environment," Phys. Rev. Lett. 103, 110402 (2009).

[10] C. J. Riedel and W. H. Zurek, "Quantum darwinism in an everyday environment: Huge redundancy in scattered photons," Phys. Rev. Lett. 105, 020404 (2010).

[11] C. J. Riedel, W. H. Zurek, and M. Zwolak, "The rise and fall of redundancy in decoherence and quantum dar- winism," New J. Phys. 14, 083010 (2012).

[12] F. Galve, R. Zambrini, and S. Maniscalco, "Nonmarkovianity hinders quantum darwinism," Sci. Rep. 6, 19607 (2015).

[13] N. Balaneskovic, "Random unitary evolution model of quantum darwinism with pure decoherence," (2015), arXiv:1510.02386, 1510.02386.

[14] J. Tuziemski and J. K. Korbicz, "Dynamical objectivity in quantum brownian motion," Europhys. Lett. 112, 40008 (2015).

[15] J. Tuziemski and J. K. Korbicz, "Objectivisation in simplified quantum brownian motion models," Photonics 2, 228 (2015).

[16] J. Tuziemski and J. K. Korbicz, "Analytical studies of spectrum broadcast structures in quantum brownian motion," J. Phys. A: Math. Theor. 49, 445301 (2016).

[17] N. Balaneskovic and M. Mendler, "Dissipation, dephasing and quantum darwinism in qubit systems with random unitary interactions," Eur. Phys. J. D 70, 177 (2016).

[18] A. Lampo, J. Tuziemski, M. Lewenstein, and J. K. Korbicz, "Objectivity in the non-markovian spin-boson model," Phys. Rev. A 96, 012120 (2017).

[19] G. Pleasance and B. M. Garraway, "Application of quantum darwinism to a structured environment," Phys. Rev. A 96, 062105 (2017).

[20] F. G. S. L. Brandão, M. Piani, and P. Horodecki, "Generic emergence of classical features in quantum darwinism," Nature Commun. 6, 7908 (2015).

[21] M. A. Nielsen and I. L. Chuang, Quantum Computation and Quantum Information: 10th Anniversary Edition, 10th ed. (Cambridge University Press, New York, NY, USA, 2011). 
[22] S. Pirandola, R. Laurenza, C. Ottaviani, and L. Banchi, "Fundamental limits of repeaterless quantum communications," Nature Communications 8, 15043 EP (2017).

[23] M. E. Shirokov, "On the energy-constrained diamond norm and its application in quantum information theory," Problems of Information Transmission 54, 20 (2018).

[24] A. Winter, "Energy-constrained diamond norm with applications to the uniform continuity of continuous variable channel capacities," (2017), arXiv:1712.10267, 1712.10267.

[25] In this paper the number operator is identified with the energy, but this is not always the case, for example in non-linear or non-harmonic (e.g., Kerr-like) processes.

[26] See Supplemental Material [EPAPS Url] for proofs and technical details, including additional Refs. [27-35].

[27] J. Watrous, "Notes on super-operator norms induced by schatten norms," arXiv preprint quant-ph/0411077 (2004).

[28] N. Killoran and N. Lütkenhaus, "Strong quantitative benchmarking of quantum optical devices," Physical Review A 83, 052320 (2011).

[29] A. Winter, "Coding theorem and strong converse for quantum channels," IEEE Transactions on Information Theory 45, 2481 (1999).

[30] T. Ogawa and H. Nagaoka, "Making good codes for classical-quantum channel coding via quantum hypothesis testing," IEEE Transactions on Information Theory 53, 2261 (2007).

[31] R. Bhatia, Matrix analysis, Vol. 169 (Springer Science \& Business Media, 2013).
[32] R. Horodecki, P. Horodecki, and M. Horodecki, "Quantum $\alpha$-entropy inequalities: independent condition for local realism?" Physics Letters A 210, 377 (1996).

[33] M. A. Nielsen and J. Kempe, "Separable states are more disordered globally than locally," Physical Review Letters 86, 5184 (2001).

[34] A. Winter, "Tight uniform continuity bounds for quantum entropies: conditional entropy, relative entropy distance and energy constraints," Communications in Mathematical Physics 347, 291 (2016).

[35] H. Qi, M. M. Wilde, and S. Guha, "Thermal states minimize the output entropy of single-mode phase-insensitive gaussian channels with an input entropy constraint," arXiv preprint arXiv:1607.05262 (2016).

[36] M. Horodecki, P. W. Shor, and M. B. Ruskai, "Entanglement breaking channels," Reviews in Mathematical Physics 15, 62917641 (2003).

[37] J. Eisert, C. Simon, and M. B. Plenio, "On the quantification of entanglement in infinite-dimensional quantum systems," Journal of Physics A: Mathematical and General 35, 3911 (2002).

[38] M. Keyl, D. Schlingemann, and R. F. Werner, "Infinitely entangled states," arXiv preprint quant-ph/0212014 (2002).

[39] G. Adesso, S. Ragy, and A. R. Lee, "Continuous variable quantum information: Gaussian states and beyond," Open Syst. Inf. Dyn. 21, 1440001 (2014).

[40] S. M. Barnett and P. M. Radmore, Methods in Theoretical Quantum Optics, Vol. 15 (Oxford University Press, 2002). 